\title{
Reversal of direct oral anticoagulants
}

This article was published in the following Dove Press journal:

Vascular Health and Risk Management

19 July 2017

Number of times this article has been viewed

\section{Mosaad Almegren}

Department of Medicine, Al Imam Mohammad Ibn Saud Islamic University, Riyadh, Kingdom of Saudi Arabia
Correspondence: Mosaad Almegren Department of Medicine, College of Medicine, Al Imam Mohammad Ibn Saud Islamic University, PO Box 7544, Othman Bin Affan Road, Al Nada, Riyadh I I432, Kingdom of Saudi Arabia

Tel +966 I 12037100

Fax +966 I 12037108

Email mosaad966@gmail.com
Abstract: Reversal agents for direct oral anticoagulants (DOACs), including factor X inhibitors and direct thrombin inhibitors, are a major concern in clinical practice. After DOACs were introduced and became widely used as an alternative for vitamin $\mathrm{K}$ antagonists in the management of venous thromboembolism and nonvalvular atrial fibrillation, the need for effective reversal agents has increased, particularly for life-threatening bleeding episodes related to DOACs or to reverse medication effects during urgent interventions. In the absence of specific reversal agents, prothrombin complex concentrate (PCC) and activated PCC are reasonable options to reverse bleeding associated with DOACs. However, high-quality clinical evidence is lacking. Idarucizumab is the only agent approved by the US Food and Drug Administration to reverse the effects of dabigatran; andexanet alfa and ciraparantag are also under evaluation as reversal agents for DOACs. This review summarizes the current evidence for nonspecific and specific reversal of DOACs.

Keywords: idarucizumab, andexanet alfa, ciraparantag, direct oral anticoagulant, reversal agents

\section{Introduction}

Direct oral anticoagulants (DOACs) have been licensed for use in the acute treatment and long-term prevention of venous thromboembolism (VTE) as well as stroke prevention in patients with nonvalvular atrial fibrillation (AF). ${ }^{1}$ DOACs include the direct thrombin inhibitors such as dabigatran and the factor $\mathrm{Xa}$ inhibitors such as rivaroxaban, apixaban, and edoxaban. Vitamin $\mathrm{K}$ antagonists (VKAs) such as warfarin and acenocoumarol are an option in VTE prevention and stroke prevention in patients with AF; however, there are many limitations to the use of VKAs: the need for parenteral anticoagulation overlap for the first few days of VTE treatment until the therapeutic international normalized ratio (INR) is achieved, the need for routine clinic visits for INR checks, concerns about food and drug interactions, and the need for perioperative bridging with parenteral anticoagulation. ${ }^{2}$ DOACs mediate their effects by direct inhibition of the coagulation factors. In general, DOACs do not require regular coagulation monitoring because these agents have predictable pharmacokinetics with fewer drug interactions and a shorter half-life that leads to rapid elimination from the body. Furthermore, these advantages are not at the expense of efficacy or safety compared with VKAs, and Phase III clinical trials of each of these drugs have shown at least similar outcomes of VTE recurrence prevention, stroke prevention in nonvalvular AF, or thromboprophylaxis after knee and hip joint arthroplasty with lower risks of intracranial bleeding. ${ }^{3,4}$ VKAs act by inhibiting the synthesis of vitamin K-dependent factors 
(II, VII, IX, X), and vitamin $\mathrm{K}$ can be used for the reversal of the VKA effects in cases of bleeding. However, immediate reversal can be achieved with prothrombin complex concentrate (PCC) or fresh frozen plasma. ${ }^{5,6}$ Unlike VKAs, the main limitation of DOACs is the lack of available specific reversal agents. Thus, specific reversal agents for DOACs are urgently needed especially in cases of emergency surgery or life-threatening bleeding.

The nonspecific agents include PCC, recombinant activated factor VII (rFVIIa), and activated PCC (aPCC), as well as hemodialysis for a dabigatran overdose. Specific antidotes include idarucizumab, andexanet alfa, and ciraparantag (PER-977).

This brief review focuses on the currently available data on DOAC reversal, including the nonspecific and specific agents.

\section{Nonspecific reversals}

The data about the efficacy of nonspecific reversal agents in DOAC-associated bleeding are scarce. However, the use of nonspecific reversal agents in life-threatening or major bleeding associated with DOACs is reasonable due to lack of specific reversal agent apart from idarucizumab for dabigatran. ${ }^{7,8}$

\section{PCC}

PCC is derived from plasma; this agent is used to reverse VKAs when there is an urgent need to correct coagulopathies associated with the administration of DOACs. ${ }^{9}$ PCC contains vitamin $\mathrm{K}$ coagulation factors: the three-factor version contains factors II, IX, and $X$ and the four-factor preparation contains factors II, VII, IX, and X along with varying quantities of protein $\mathrm{C}$ and $\mathrm{S}$. The clinical studies in humans have been limited and restricted to healthy volunteers. In three small randomized, placebo-controlled studies, PCC reversed the anticoagulation effects of rivaroxaban and edoxaban, but it did not reverse the effects in patients treated with dabigatran. ${ }^{10-12}$ In 12 healthy volunteers, six received $20 \mathrm{mg}$ rivaroxaban twice a day, or $150 \mathrm{mg}$ dabigatran twice a day in another six healthy volunteers for 2.5 days. PCC infusion $(50 \mathrm{U} / \mathrm{kg})$ corrected prolonged prothrombin time (PT) induced by rivaroxaban, while prolonged activated partial thromboplastin time (aPTT), thrombin time (TT), and ecarin clotting time (ECT) induced by dabigatran were not corrected..$^{10}$ In an open-label, parallel-group study, comparing three-factor PCC to four-factor PCC formulations in PT reversal, 35 healthy volunteers received rivaroxaban $20 \mathrm{mg}$ twice a day for 4 days followed by $50 \mathrm{U} / \mathrm{kg}$ of three- or four-factor PCC infusion. Three-factor PCC reduced mean
PT by $0.6-1$ second, whereas four-factor PCC reduced the mean PT by $2.5-3.5$ seconds. ${ }^{11}$ Based on these studies, a dose of $50 \mathrm{U} / \mathrm{kg}$ of PCC can be used to reverse the effects of DOACs in patients with severe or life-threatening bleeding. In a randomized crossover trial, 12 healthy volunteers received apixaban $5 \mathrm{mg}$ twice daily for 2.5 days followed by four-factor PCC (25 U/kg) infusion 3 hours after last dose of apixaban. ${ }^{13}$ Thrombin generation was increased by $76 \%$ at 30 minutes post four-factor PCC infusion. There were no differences in anti-Xa or partial thromboplastin time (PTT) values between four-factor PCC and placebo. ${ }^{13}$

In one meta-analysis, $1.4 \%$ of patients who received PCC for the correction of a major bleeding episode associated with warfarin developed thromboembolic events. ${ }^{14}$

\section{aPCC}

aPCC is an anti-inhibitor coagulant complex that works as a factor VIII inhibitor bypassing activity (FEIBA). ${ }^{15}$ This agent is also derived from plasma and contains activated factor VII along with the nonactivated factors II, IX, and X. It was initially developed to manage bleeding in patients with hemophilia who had inhibitors to factor VIII or IX. Recent studies have elaborated its usefulness as an off-label drug that can be used to reverse the anticoagulative effects in actively bleeding patients. Studies also suggested that aPCC has the ability to reverse bleeding associated with DOACs.

In a series of 127 patients, six patients developed spontaneous intracranial hemorrhage (ICH) secondary to DOAC use and were managed with aPCC at $50 \mathrm{U} / \mathrm{kg}$; none of these patients developed an ICH expansion or thrombotic or hemorrhagic complications. ${ }^{16}$ Marlu et al ${ }^{17}$ carried out an investigation on 10 healthy volunteers who had been randomly given dabigatran or rivaroxaban. In a dose-dependent manner, aPCC corrected the coagulation parameters that had a correlation with the generation of thrombin (endogenous thrombin potential and thrombin peak). ${ }^{17}$ However, these studies were inconclusive because there have been no concrete studies that have evaluated the effectiveness of this agent in the reversal of DOACs.

\section{rFVIla}

This factor was initially developed for the treatment of bleeding in patients with hemophilia who had inhibitors. A dose of $90 \mu \mathrm{g} / \mathrm{kg}$ has been suggested to reverse major DOAC-associated bleeding. ${ }^{8}$ However, no clinical studies have evaluated the reversal of DOAC with rFVIIa. Compared to placebo, the off-label use of rFVIIa was associated with an increased risk of arterial thromboembolic events. ${ }^{18}$ 


\section{Hemodialysis for dabigatran}

Hemodialysis has been used in the reversal of dabigatranassociated life-threatening bleeding. ${ }^{19}$ Dabigatran has a large fraction that is not protein bound and a lipophilic structure which makes it easily dialyzable. Hemodialysis removes up to $68 \%$ of dabigatran..$^{20}$ Apixaban and rivaroxaban are not dialyzable because they are highly protein bound. ${ }^{6}$ Edoxaban is $\sim 50 \%$ bound to proteins. However, in an open-label, Phase I, randomized trial conducted to evaluate the pharmacokinetics of edoxaban in 10 patients on hemodialysis, edoxaban $15 \mathrm{mg}$ was given to the patients 2 hours prior to dialysis or in between dialysis sessions. Mean maximum plasma concentrations were comparable in both groups. ${ }^{21}$ This study showed that hemodialysis was not an effective method for removing edoxaban from the system. ${ }^{21}$

\section{Specific reversal agents}

The lack of specific antidotes has been the major limitation against the utilization of DOACs, which encourages physicians and patients to give higher preference to VKAs. The abovementioned data about nonspecific reversal of DOACs do not provide enough evidence to support use in DOACassociated bleeding. Thus, several new specific reversal agents such as idarucizumab, andexanet alfa, and ciraparantag (PER-977) have recently been developed.

\section{Idarucizumab}

Idarucizumab is derived from humanized monoclonal antibody segments. It binds to the benzamide moiety in the region between the heavy- and light-chain variable domains via the salt bridge and the hydrophobic and hydrogen bonding. This results in an irreversible affinity that is $>350$-fold stronger than the one that dabigatran has toward thrombin and thus rapidly neutralizes the anticoagulant effects that the drug generates. ${ }^{22}$ This high affinity ensures that the drug binds specifically to dabigatran and not to other thrombin-associated substrates such as von Willebrand factor, fibrinogen, protein C, and factors II, VIII, and XIII; this also ensures that platelet aggregation is not interrupted. As with dabigatran, idarucizumab is excreted by the kidney. In patients with normal renal function, idarucizumab had a half-life of 45 minutes. ${ }^{23}$

The potency of this drug has been tested in Phase I and II clinical trials and has been shown to be highly effective in the reversal of dabigatran-associated bleeding. ${ }^{23,24}$ Idarucizumab can reverse the aPTT, TT, diluted TT (dTT), and ECT in a dose-dependent fashion in healthy volunteers and patients with renal impairment. ${ }^{23,24}$ In healthy male volunteers, the administration of idarucizumab had no effect on the coagulation profile, suggesting that idarucizumab was likely a non-prothrombotic agent. ${ }^{25}$

The RE-VERSE AD (Reversal Effects of Idarucizumab on Active Dabigatran) is an ongoing Phase III prospective trial investigating two groups of dabigatran-treated patients: group A patients required reversal because of severe bleeding and group B patients required reversal because of an urgent intervention. ${ }^{26}$ Idarucizumab was administered at a dose of $5 \mathrm{~g}$ divided into two $2.5 \mathrm{~g}$ intravenous boluses over 5-10 minutes. ${ }^{26}$ The primary outcome is the maximum percentage reversal of the dabigatran anticoagulant effects within 4 hours of the intravenous idarucizumab boluses and is based on central laboratory measurements of the dTT or ECT. The secondary outcome is the time to bleeding cessation in group $\mathrm{A}$ and the time to intraoperative hemostasis in group B. The analysis of the first 90 patients investigated in RE-VERSE $\mathrm{AD}$ consisted of 51 patients in group A and 39 patients in group B. The median maximum percentage reversal in both groups was $100 \%$. Idarucizumab reversed the coagulation effects in $88 \%-98 \%$ patients with elevated ECT and dTT. The median time to bleeding cessation was 11.4 hours in 35 of the patients in group A. The intraoperative hemostasis was determined to be normal in 33 of the patients from group $\mathrm{B}$ and mild to moderately abnormal in three other patients. There was one thrombotic event within 72 hours of the idarucizumab administration, and four events occurred later. ${ }^{26}$

A follow-up analysis of RE-VERSE AD includes 494 patients, 298 in group A (patients with severe bleeding) and 196 in group B (patients requiring urgent intervention). ${ }^{27}$ The median maximum percentage reversal within 4 hours in both groups was $100 \%$ for both ECT and dTT. Idarucizumab reversed prolonged ECT within 4 hours in $81.5 \%$ of patients in group A and $83.5 \%$ in group B. Normalization of dTT occurred within 4 hours in $98.7 \%$ of patients in group A and $98.6 \%$ in group B. The median time to bleeding cessation in group A determined by an investigator was 3.5 hours in 97 patients with gastrointestinal bleeding and 4.5 hours in 61 patients with non-gastrointestinal and non-intracranial bleeding. There were 35 thrombotic events occurred in 31 of $494(6.3 \%)$ patients at 90 days. ${ }^{27}$

In October 2015, idarucizumab received US Food and Drug Administration (FDA) approval for use in patients with dabigatran-associated bleeding in cases of emergency procedures or of life-threatening or uncontrolled bleeding (Table 1). 
Table I Summary of specific reversal agents for DOACs

\begin{tabular}{|c|c|c|c|}
\hline & Idarucizumab & Andexanet alfa & Ciraparantag \\
\hline Target & Thrombin inhibitor (dabigatran) & $\begin{array}{l}\text { Direct factor Xa inhibitors (rivaroxaban, } \\
\text { apixaban, edoxaban), LMWH, } \\
\text { fondaparinux }\end{array}$ & $\begin{array}{l}\text { Dabigatran, rivaroxaban, apixaban, } \\
\text { edoxaban, LMWH, fondaparinux }\end{array}$ \\
\hline Mechanism of action & Noncompetitive binding to dabigatran & $\begin{array}{l}\text { Binding to direct and indirect factor } \mathrm{Xa} \\
\text { inhibitors }\end{array}$ & $\begin{array}{l}\text { Noncovalent hydrogen bonding } \\
\text { binds to the target drug }\end{array}$ \\
\hline Onset of action & Immediate & $2-5$ minutes & 10 minutes \\
\hline Administration and dose & $\begin{array}{l}5 \mathrm{~g} \text { intravenously as two doses of } 2.5 \mathrm{~g} \\
\text { each, not more than } 15 \text { minutes apart* }\end{array}$ & $\begin{array}{l}400-800 \mathrm{mg} \text { intravenous bolus followed } \\
\text { by } 4-8 \mathrm{mg} / \mathrm{min} \text { infusion } \S\end{array}$ & $100-300 \mathrm{mg}$ intravenous bolus ${ }^{ \pm}$ \\
\hline Elimination & Renal & Not reported & Not reported \\
\hline
\end{tabular}

Notes: *FDA-approved dose. ${ }^{\S}$ Doses used in clinical trials but not approved. ${ }^{ \pm}$Dose used in Phase I clinical trial.

Abbreviations: DOACs, direct oral anticoagulants; FDA, US Food and Drug Administration; LMWH, low molecular weight heparin.

\section{Andexanet alfa}

Andexanet alfa is a specific antidote for direct (rivaroxaban, apixaban, and edoxaban) and indirect factor Xa inhibitors (low molecular weight heparin and fondaparinux). It has the potential to reverse the anticoagulant effects that specifically target activated factor X since it is a modified human recombinant form of factor $\mathrm{X}$. This drug binds to factor $\mathrm{X}$ inhibitors with the same affinity as the activated native factor $\mathrm{X} .{ }^{28}$

In animal bleeding models, andexanet reversed the anticoagulation effects and reduced blood loss. ${ }^{29}$ In Phase II studies in healthy volunteers, the researchers found that an intravenous andexanet bolus reversed the effects of factor Xa inhibitors (rivaroxaban, apixaban, and edoxaban) in a dose-dependent manner. ${ }^{28,30}$

Based on these data, a two-part randomized placebocontrolled trial, ANNEXA (Andexanet Alfa, a Novel Antidote to the Anticoagulation Effects of FXA Inhibitors), was performed in healthy volunteers treated with apixaban (ANNEXA-A) or rivaroxaban (ANNEXA-R) ${ }^{28} \mathrm{~A}$ total of 145 participants on apixaban or rivaroxaban were assigned to either andexanet or placebo. Each trial was conducted in two parts. In ANNEXA-A, an intravenous bolus of andexanet was given at a dose of $400 \mathrm{mg}(30 \mathrm{mg} / \mathrm{min})$ in part 1 or a $400 \mathrm{mg}$ intravenous bolus followed by a continuous infusion for 120 minutes at a dose of $4 \mathrm{mg} / \mathrm{min}$ was given in part 2. In ANNEXA-R, an intravenous bolus of andexanet was given at a dose of $800 \mathrm{mg}$ in part 1 or a $800 \mathrm{mg}$ intravenous bolus followed by a continuous infusion for 120 minutes at a dose of $8 \mathrm{mg} / \mathrm{min}$ was given in part 2. Higher doses of andexanet were used to reverse rivaroxaban compared to the doses used for apixaban because of the higher plasma concentration and larger volume distribution of rivaroxaban. The primary end point was the percent change in factor Xa inhibitor activity from the baseline to nadir. An andexanet bolus reduced antifactor Xa levels within 2-5 minutes in both trials by $>90 \%$ compared to $\sim 20 \%$ with placebo $(P<0.001)$. Within 2 hours, the anti-factor Xa levels returned to the levels seen in the placebo group; however, the reversal effect was maintained when andexanet was given as a bolus and followed by infusion. No thrombotic or serious events were reported. ${ }^{28}$

The efficacy and safety of andexanet alfa are under evaluation in an ongoing Phase IIIb/IV study (ANNEXA-4) for patients with severe bleeding taking rivaroxaban, apixaban, or edoxaban. In a preliminary report of the ANNEXA-4 trial, patients who had taken apixaban or rivaroxaban for $>7$ hours before the administration of andexanet alfa received a dose of $400 \mathrm{mg}$ bolus followed by $480 \mathrm{mg}$ infusion over 2 hours, ${ }^{31}$ and patients who had taken enoxaparin, edoxaban, or rivaroxaban within 7 hours or at an unknown time received a dose of $800 \mathrm{mg}$ bolus followed by $960 \mathrm{mg}$ infusion over 2 hours. An independent committee determined the hemostatic efficacy on the basis of predefined criteria. Of 47 patients in efficacy population, 37 had excellent or good hemostasis (79\%; 95\% CI, $64 \%-89 \%)$. Of 67 patients in safety population, 12 had thrombotic events (18\%) within 30 days after andexanet alfa was administered. The thromboses included one myocardial infarction, five strokes, seven deep vein thrombosis, and one pulmonary embolism. ${ }^{31}$

\section{Ciraparantag (aripazine; PER977)}

Ciraparantag is a synthetic water-soluble molecule that has been developed to form strong noncovalent bonds and large complex molecules that bind heparin, direct factor Xa inhibitors, and thrombin inhibitors. ${ }^{32}$ In animal bleeding models, reversal of the anticoagulant effect was observed by a reduction in blood loss. Ciraparantag reversed the effects of direct factor Xa inhibitor in a dose-dependent manner in ex vivo human blood without an observed procoagulant effect. ${ }^{33}$

In a Phase I study on 80 healthy volunteers, ciraparantag was administered intravenously as a single dose (100-300 mg) after a dose of edoxaban. The anticoagulation reversal was assessed by the whole blood clotting time that 
was reduced to $\sim 10 \%$ of baseline in 10 minutes with 24 hours of sustained effect. ${ }^{34}$

Ciraparantag is under evaluation in a Phase II clinical trial investigating reanticoagulation with edoxaban and a second reversal with ciraparantag. ${ }^{35}$

\section{Conclusion}

DOACs carry a risk of bleeding as with all antithrombotic therapies. Nonspecific reversal agents have been used in clinical practice, mainly in DOAC-related major bleeding, and to reverse the effects in urgent interventions. Idarucizumab is an FDA-approved agent to reverse dabigatran effects. Currently, andexanet alfa and ciraparantag are under development as specific reversal agents. In DOAC-related bleeding, the improvement from specific reversal agents still needs to be evaluated.

\section{Disclosure}

The author reports no conflicts of interest in this work.

\section{References}

1. Weitz JI. Anticoagulation therapy in 2015: where we are and where we are going. J Thromb Thrombolysis. 2015;39(3):264-272.

2. Gómez-Outes A, Suárez-Gea ML, Calvo-Rojas G, et al. Discovery of anticoagulant drugs: a historical perspective. Curr Drug Discov Technol. 2012;9(2):83-104

3. Chai-Adisaksopha C, Hillis C, Isayama T, Lim W, Iorio A, Crowther M. Mortality outcomes in patients receiving direct oral anticoagulants: a systematic review and meta-analysis of randomized controlled trials. J Thromb Haemost. 2015;13(11):2012-2020.

4. Kearon C, Akl EA, Ornelas J, et al. Antithrombotic therapy for VTE disease: CHEST guideline and expert panel report. Chest. 2016;149(2):315-335.

5. Fasco MJ, Principe LM. R- and S-Warfarin inhibition of vitamin K and vitamin K 2,3-epoxide reductase activities in the rat. J Biol Chem. 1982;257(9):4894-4901.

6. Ageno W, Gallus AS, Wittkowsky A, Crowther M, Hylek EM, Palareti G. Oral anticoagulant therapy: antithrombotic therapy and prevention of thrombosis, 9th ed: American College of Chest Physicians evidencebased clinical practice guidelines. Chest. 2012;141(2 Suppl):44S-88S

7. Ageno W, Büller HR, Falanga A, et al. Managing reversal of direct oral anticoagulants in emergency situations. Anticoagulation Education Task Force White Paper. Thromb Haemost. 2016;116(6):1003-1010.

8. Heidbuchel H, Verhamme P, Alings M, et al. Updated European Heart Rhythm Association Practical Guide on the use of nonvitamin K antagonist anticoagulants in patients with non-valvular atrial fibrillation. Europace. 2015;17(10):1467-1507.

9. Wann LS, Curtis AB, Ellenbogen KA, et al. 2011 ACCF/AHA/HRS focused update on the management of patients with atrial fibrillation (update on dabigatran): a report of the American College of Cardiology Foundation/American Heart Association Task Force on practice guidelines. J Am Coll Cardiol. 2011;57(11):1330-1337.

10. Eerenberg ES, Kamphuisen PW, Sijpkens MK, Meijers JC, Buller HR, Levi M. Reversal of rivaroxaban and dabigatran by prothrombin complex concentrate: a randomized, placebo-controlled, crossover study in healthy subjects. Circulation. 2011;124(14):1573-1579.

11. Levi M, Moore KT, Castillejos CF, et al. Comparison of three-factor and four-factor prothrombin complex concentrates regarding reversal of the anticoagulant effects of rivaroxaban in healthy volunteers. JThromb Haemost. 2014;12(9):1428-1436.
12. Zahir H, Brown KS, Vandell AG, et al. Edoxaban effects on bleeding following punch biopsy and reversal by a 4-factor prothrombin complex concentrate. Circulation. 2015;131(11):82-90.

13. Nagalla S, Thomson L, Oppong Y, Bachman B, Chervoneva I, Kraft WK. Reversibility of apixaban anticoagulation with a four-factor prothrombin complex concentrate in healthy volunteers. Clin Transl Sci. 2016;9(3):176-180.

14. Dentali F, Marchesi C, Pierfranceschi MG, et al. Safety of prothrombin complex concentrates for rapid anticoagulation reversal of vitamin $\mathrm{K}$ antagonists. A meta-analysis. Thromb Haemost. 2011;106(3):429-438.

15. Aledort LM. Comparative thrombotic event incidence after infusion of recombinant factor VIIa versus factor VIII inhibitor bypass activity. J Thromb Haemost. 2004;2(10):1700-1708.

16. Dibu JR, Weimer JM, Ahrens C, Manno E, Frontera JA. The role of FEIBA in reversing novel oral anticoagulants in intracerebral hemorrhage. Neurocrit Care. 2016;24(3):413-419.

17. Marlu R, Hodaj E, Paris A, Albaladejo P, Cracowski JL, Pernod G. Effect of non-specific reversal agents on anticoagulant activity of dabigatran and rivaroxaban. A randomised crossover ex vivo study in healthy volunteers. Thromb Haemost. 2012;108(2):217-224.

18. Levi M, Levy JH, Andersen HF, TruloffD. Safety of recombinant activated factor VII in randomized clinical trials. N Engl J Med. 2010;363(19): 1791-1800.

19. Warkentin TE, Margetts P, Connolly SJ, Lamy A, Ricci C, Eikelboom JW. Recombinant factor VIIa (rFVIIa) and hemodialysis to manage massive dabigatran-associated postcardiac surgery bleeding. Blood. 2012;119(9):2172-2174.

20. Stangier J, Rathgen K, Stahle H, et al. Influence of renal impairment on the pharmacokinetics and pharmacodynamics of oral dabigatran etexilate: an open-label, parallel-group, single-centre study. Clin Pharmacokinet. 2010;49:259-268.

21. Parasrampuria DA, Marbury T, Matsushima N, et al. Pharmacokinetics, safety, and tolerability of edoxaban in end-stage renal disease subjects undergoing haemodialysis. Thromb Haemost. 2015;113(4): 719-727.

22. Schiele F, van Ryn J, Canada K, et al. A specific antidote for dabigatran: functional and structural characterization. Blood. 2013;121(18): 3554-3562.

23. Glund S, Stangier J, Schmohl M, et al. Safety, tolerability, and efficacy of idarucizumab for the reversal of the anticoagulant effect of dabigatran in healthy male volunteers: a randomised, placebo-controlled, doubleblind phase 1 trial. Lancet. 2015;386(9994):680-690.

24. Glund S, Stangier J, Schmohl M, et al. Idarucizumab, a specific antidote for dabigatran: immediate, complete and sustained reversal of dabigatran induced anticoagulation in elderly and renally impaired subjects. Blood. 2014;124(21):344

25. Glund S, Moschetti V, Norris S, et al. A randomised study in healthy volunteers to investigate the safety, tolerability and pharmacokinetics of idarucizumab, a specific antidote to dabigatran. Thromb Haemost. 2015;113(5):943-951.

26. Pollack CV Jr, Reilly PA, Eikelboom J, et al. Idarucizumab for dabigatran reversal. $N$ Engl J Med. 2015;373(6):511-520.

27. Pollack CV. Idarucizumab for dabigatran reversal: updated results of the RE-VERSE AD Study. Presented at 2016 American Heart Association Scientific Sessions 2016; New Orleans, LA.

28. Siegal DM, Curnutte JT, Connolly SJ, et al. Andexanet alfa for the reversal of factor Xa inhibitor activity. $N$ Engl J Med. 2015;373(25): 2413-2424.

29. Hollenbach S, Lu G, DeGuzman F, et al. Abstract 14657: andexanet-alfa and PER977 (arapazine) correct blood loss in a rabbit liver laceration model-only andexanet reverses markers of fXa-mediated anticoagulation. Circulation. 2014;130(Suppl 2):A14657.

30. Crowther M, Levy GG, Lu G, et al. A phase 2 randomized, double-blind, placebo-controlled trial demonstrating reversal of edoxaban-induced anticoagulation in healthy subjects by andexanet alfa (PRT064445), a universal antidote for factor Xa (fXa) inhibitors. Blood. 2014;124(21): 4269 . 
31. Connolly SJ, Milling TJ Jr, Eiklboom JW, et al; the ANNEXA-4 Investigators. Andexanet alfa for acute major bleeding associated with factor Xa Inhibitors. $N$ Engl J Med. 2016;375(12):1131-1141.

32. Greinacher A, Thiele T, Selleng K. Reversal of anticoagulants: an overview of current developments. Thromb Haemost. 2015;113(5):931-942.

33. Laulicht B, Bakhru S, Lee C, et al. Small molecule antidote for anticoagulants. Circulation. 2012;126(suppl 21):A11395.
34. Ansell JE, Bakhru SH, Laulicht BE, et al. Use of PER977 to reverse the anticoagulant effect of edoxaban. $N$ Engl J Med. 2014;371(22): 2141-2142.

35. Perosphere, Inc. Study of PER977 administered to subjects with steady state edoxaban dosing and re-anticoagulation with edoxaban. Available from: https://www.clinicaltrials.gov/ct2/show/NCT02207257. NLM identifier: NCT02207257. Accessed April 3, 2017.

\section{Publish your work in this journal}

Vascular Health and Risk Management is an international, peerreviewed journal of therapeutics and risk management, focusing on concise rapid reporting of clinical studies on the processes involved in the maintenance of vascular health; the monitoring, prevention and treatment of vascular disease and its sequelae; and the involvement of metabolic disorders, particularly diabetes. This journal is indexed on PubMed Central and MedLine. The manuscript management system is completely online and includes a very quick and fair peer-review system, which is all easy to use. Visit http://www.dovepress.com/ testimonials.php to read real quotes from published authors. 